\title{
Nano-thermometer Based on Resonant Tunneling Diodes: from Cryogenic to Room Temperatures
}

Andreas Pfenning, ${ }^{\dagger}$ Fabian Hartmann, ${ }^{\dagger}$ Mariama Rebello Sousa Dias, ${ }^{\dagger}$ Leonardo

Kleber Castelano,$^{\ddagger}$ Christoph Süßmeier, ${ }^{\dagger}$ Fabian Langer, ${ }^{\dagger}$ Sven Höfling, ${ }^{\dagger}, \uparrow$ Martin Kamp, ${ }^{\dagger}$ Gilmar Eugenio Marques,,$^{\ddagger}$ Lukas Worschech, ${ }^{*} \dagger$ and Victor Lopez-Richard*,‡

†Technische Physik, Physikalisches Institut, Universität Würzburg and Wilhelm Conrad Röntgen Research Center for Complex Material Systems, Am Hubland, D-97074 Würzburg, Germany

†Departamento de Física, Universidade Federal de São Carlos, 13565-905 São Carlos, SP, Brazil

ๆSUPA, School of Physics and Astronomy, University of St Andrews, St Andrews, KY16 9SS, United Kingdom

E-mail: lukas.worschech@uni-wuerzburg.de; vlopez@df.ufscar.br

Keywords: Resonant Tunneling Diode, Thermometer. Keywords: Resonant Tunneling Diode, Thermometer.

\begin{abstract}
Sensor miniaturization together with broadening temperature sensing range are fundamental challenges in nano-thermometry. By exploiting a large temperature dependent screening effect observed in a resonant tunneling diode in sequence with a GaInNAs/GaAs quantum well, we present a low dimensional, wide range, and high
\end{abstract}


sensitive nano-thermometer. This sensor shows a large threshold voltage shift of the bistable switching of more than $4.5 \mathrm{~V}$ for a temperature raise from $4.5 \mathrm{~K}$ to $295 \mathrm{~K}$, with a linear voltage-temperature response of $19.2 \mathrm{mV} / \mathrm{K}$, and a precision in the $\mathrm{mK}$ range. Also, monitoring the electroluminescence emission spectrum, an optical readout control of the thermometer is provided. The combination of electrical and optical read-outs together with the sensor architecture excel the device as a thermometer with the capability of non-invasive temperature sensing, high local resolution, and sensitivity.

\section{Introduction}

Thermometry at the nanoscale attracts considerable interest ${ }^{1,2}$ and the demand for accurate temperature mapping spans from microcircuits in solid-state devices $\stackrel{3}{3} \sim{ }_{\sim}$ to living cells in biological systems. ${ }^{5,6}$ Specifically at the nanoscale, temperature differences of a few Kelvin can alter the device performance significantly. ${ }^{7,8}$ Additionally, heat dissipation and thermal transport properties may be significantly different in low dimensions compared to bulk-like systems. 9,11 9-11 Therefore, it is crucial to have precise thermometers with high local resolution. In this field, different types of thermometers exist which exploit different thermometric effects. For instance, one can cite the Seebeck voltage in thermocouples, ${ }^{3}$ thermal expansion of liquids in carbon nanotubes, ${ }^{12}$ and the temperature-dependent Johnson noise ${ }^{13}$ and shot noise in tunnel junctions ${ }^{14}$ which usually require advanced read-out schemes.

The sensor concept proposed here can be achieved by electronic structure and doping engineering of resonant tunneling diodes (RTDs) in sequence with a heterojunction interface and is provided by a wide and deep, and heavily n-doped quantum well. At the junction, a temperature-dependent electric field screening due to accumulated electrons at the interface, leads to a large threshold voltage shift of the RTD bistable transition with a linear voltagetemperature response. 


\section{Design and fabrication}

The RTD was grown by molecular beam epitaxy in the following way. On top of an $n$-doped GaAs source layer, an undoped double barrier structure (DBS) was realized, which consists of two $3 \mathrm{~nm}$ thick $\mathrm{Al}_{0.6} \mathrm{Ga}_{0.4}$ As barriers sandwiching a $4 \mathrm{~nm}$ thick GaAs quantum well. At the source and before the DBS, $300 \mathrm{~nm}$ GaAs were deposited with doping concentration from $n=10^{18} \mathrm{~cm}^{-3}$ to $10^{17} \mathrm{~cm}^{-3}$ followed by $l_{a}=15 \mathrm{~nm}$ undoped GaAs. The DBS thickness is $l_{d b}=10 \mathrm{~nm}$. After the DBS, a $5 \mathrm{~nm}$ thick undoped GaAs spacer was grown followed by an $l_{N}=160 \mathrm{~nm}$ thick $\mathrm{Ga}_{1-x} \operatorname{In}_{x} \mathrm{~N}_{y} \mathrm{As}_{1-y}$ layer. The $\mathrm{N}$ and In contents, $x=11 \%$ and $y=4 \%$, ensure a lattice-matched growth to GaAs with a band gap energy of $E_{g}=0.95 \mathrm{eV} .{ }^{15}$ The corresponding conduction band offset of the GaInNAs/GaAs interface is $\approx 0.38 \mathrm{eV}$. Finally, a $500 \mathrm{~nm}$ thick $n$-doped GaAs drain contact layer was grown on top. After the growth process, RTD mesas with diameters ranging from 1 to $12 \mu \mathrm{m}$ were processed by electron beam lithography and dry chemical etching techniques. The top ring-shaped contact is composed of $\mathrm{Ti} / \mathrm{Pt} / \mathrm{Au}$ and the bottom contact, of alloyed $\mathrm{AuGe} / \mathrm{Ni} / \mathrm{Au}$.

Additionally, a reference structure was grown and studied in analogy to the RTD under characterization for which the GaInNAs region was replaced by GaAs leaving everything else unaltered. It allows contrasting the main thermometric properties of the sensor.

\section{Results and discussion}

A schematic illustration of the RTD thermometer is shown in 1A. The corresponding conduction (CB) and valence band (VB) profiles as well as the charge carrier densities are represented in $1 \mathrm{~B}$ and $\mathrm{C}$. Electrons injected from the source can tunnel resonantly through the DBS whenever their energy is equal to the one of a quantum well layer subband. The

current $I$ depends on the transmission probability and the electron supply function, ${ }^{16,17}$ while the RTD voltage drop, $V_{r t d}$, is mainly determined by the arrangement of accumulated electron and ionized donor densities. ${ }^{18}$ Two electron densities, $n_{a c}$ and $n_{i}$, are formed due 


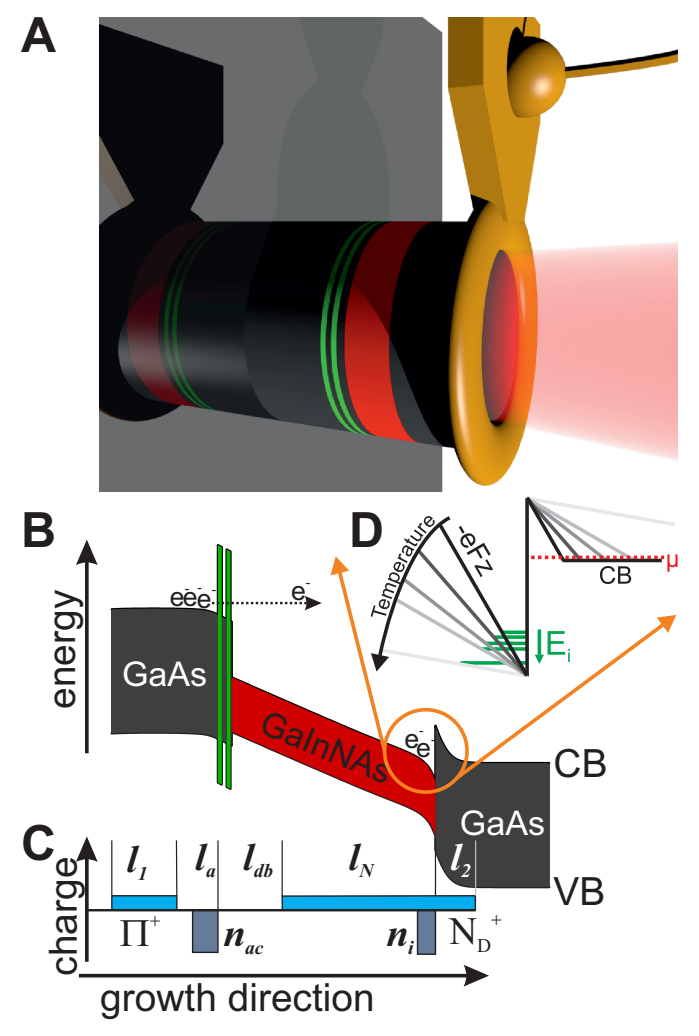

Figure 1: (A) Scheme of the working principle of the RTD nano-thermometer. (B) Conduction and valence band profiles with electrons injected from the source. (C) Charge density profiles: the RTD voltage drop $V_{r t d}(T)$ results from the arrangement of the 2-D sheet electron densities $n_{i}, n_{a c}$ and the $3-\mathrm{D}$ donor densities $\Pi^{+}$and $N_{D}^{+}$along the structure. (D) Temperature evolution of the GaInNAs/GaAs interface with the energy of the first quantized state $E_{1}$, the chemical potential $\mu$ and the corresponding potential $-|e| F z$. 
to electrons at the source and at the GaInNAs/GaAs interface. The first one is formed at a distance $l_{a}$, and the second one, at $l_{N}$ from the DBS, as indicated in $1 \mathrm{C}$. The donor density at the source, $\Pi^{+}$, is uniformly distributed along the distance $l_{1}$ of the doped layer, a length $l_{a}$ apart from the DBS. The density $N_{D}^{+}$spreads within the GaInNAs layer and the GaAs drain side of the interface with lengths $l_{N}$ and $l_{2}$, respectively. 1D shows schematically the band profile at the GaInNAs/GaAs interface for different temperatures. As one can see later, this temperature-dependent interplay between the accumulated electron density and the electric field alters the resonance bias voltage of the RTD.

\section{Thermometric transport response}

Thermometric transport response. The experiments were performed in the following way: for temperatures between $4.5 \mathrm{~K}$ and $295 \mathrm{~K}$, the current $I$ through the RTD was measured by sweeping the voltage, $V_{r t d}$, from $0 \mathrm{~V}$ to $11 \mathrm{~V}$ and back to $0 \mathrm{~V}$. $2 \mathrm{~A}$ shows the corresponding RTD I-V characteristics for various temperatures ranging from $T=4.5 \mathrm{~K}$ up to $T=295 \mathrm{~K}$. At room temperature, the RTD is bistable with a threshold voltage for the up-sweep direction (increasing voltage) $V_{u}=6.48 \mathrm{~V}$ and $V_{d}=5.69 \mathrm{~V}$, for the down-sweep direction (decreasing voltage). The peak and valley current are $I_{p}=1.8 \mathrm{~mA}$ and $I_{v}=0.6 \mathrm{~mA}$ with a corresponding peak-to-valley current ratio (PVR) of 3.1. When the temperature is reduced from $T=295 \mathrm{~K}$ to $4.5 \mathrm{~K}$, the bistable region of the RTD I-V characteristic shifts to larger bias voltages, e.g. the up- and down-sweep thresholds are $V_{u}=10.91 \mathrm{~V}$ and $V_{d}=9.83 \mathrm{~V}$ for $T=4.5 \mathrm{~K}$.

2B shows various $\mathrm{I}-\mathrm{V}$ characteristics from $T=295 \mathrm{~K}$ to $4.5 \mathrm{~K}$ that were simulated using a modified Schulman's equation ${ }^{19}$ with a temperature dependent voltage shift $\Delta V$. The Lorentzian shaped transmission $T_{r}$ in Schulman's model does not account for different types of broadening effects, such as inelastic phonon scattering. According to Büttiker, the total width of the transmission, $\Gamma=\Gamma_{e}+\Gamma_{i}$, can be expressed as the sum of elastic $\Gamma_{e}$ and inelastic 
$\Gamma_{i}$ broadenings. The transmission function is then given by ${ }^{20}$

$$
T_{r}=\frac{\frac{1}{4} \Gamma_{e} \Gamma_{i}}{\left(E-E_{r}\right)^{2}+\frac{1}{4} \Gamma^{2}} .
$$

The electron supply function used in Schulman's equation considers emission from a flat band, that we assumed as temperature independent, using the effective mass approximation and the Fermi distribution function. The current is then given by

$$
J=\frac{\Gamma_{e} m^{*} k T}{8 \pi^{2} \hbar^{3}}\left[\tan ^{-1}\left(\frac{E_{r}-\eta V}{\Gamma_{i} / 2}\right)+\frac{\pi}{2}\right] \ln \left(\frac{e^{\frac{\eta V+E_{F}-E_{r}}{k T}}+1}{e^{\frac{-\eta V+E_{F}-E_{r}}{k T}}+1}\right)+h \cdot\left(e^{\frac{\eta V}{k T}}-1\right) .
$$

The second term on the right side of Eq. 2 accounts for the additional current of the diode form. ${ }^{20}$ The parameters used in the calculations were: $m^{*}=0.061 m_{0^{-}}$the GaAs conduction band effective mass, $E_{r}=150 \mathrm{meV}$ - the confined energy level measured from the conduction band bottom at the source, $E_{F}=50 \mathrm{meV}$ - the corresponding Fermi energy, $\Gamma_{e}=1 \mathrm{meV}$ and $\Gamma_{i}=\Gamma_{e}+2 \cdot 10^{-5} T[\mathrm{meV}]$-the elastic and inelastic broadenings, $\eta=0.04$ - the efficiency, and $h=3000$ - a correction parameter for the valley current. To account for the additional temperature dependent voltage shift $\Delta V, V$ is replaced in Eq. 2 by

$$
(V-\Delta V) \times \Theta[V-\Delta V],
$$

where $\Theta$ is the Heaviside function. The description of the voltage threshold shift, $\Delta V$, dependence on temperature will be given below. To assess the relevance of this effect, selected I-V curves from the reference sample (described in the Design and fabrication section) are shown in $2 \mathrm{C}$ for temperatures $T=295 \mathrm{~K}, 77 \mathrm{~K}$, and $4.2 \mathrm{~K}$. In contrast to the reference sample, the RTD with the GaInNAs/GaAs layer shows a large shift of the threshold voltages $V_{u}$ and $V_{d}$ with temperature.

The temperature dependence of the threshold voltages, $V_{\alpha}$, with $\alpha=t, u, d$, are plotted in $3 \mathrm{~A}, . V_{u}$ and $V_{d}$ correspond to the separation voltages of the bistable region, while $V_{t}$ is 

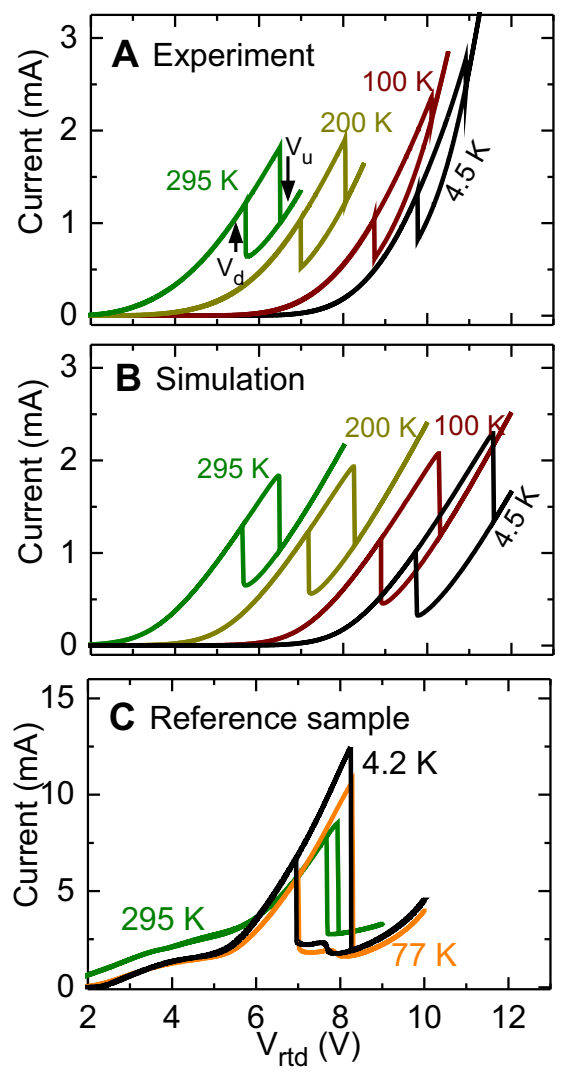

Figure 2: Temperature dependence of the current-voltage characteristics. (A) I-V curves for various temperatures from $T=4.5 \mathrm{~K}$ to $295 \mathrm{~K}$ for the sample with the GaInNAs/GaAs heterojuntion. The threshold voltages $V_{u}$ and $V_{d}$ shift to larger bias voltages, when the temperature is reduced from $T=295 \mathrm{~K}$ to $4.5 \mathrm{~K}$. (B) Simulated I-V curves for various temperatures from $T=4.5 \mathrm{~K}$ to $295 \mathrm{~K}$ using a modified Schulman's equation. (C) I-V characteristics of the reference sample show no pronounced threshold voltage shift, when the temperature changes. 
defined for a current flow, $I_{t}=100 \mu A$, i.e. $V\left(I_{t}=100 \mu A\right)=V_{t}$. All three threshold voltages show a pronounced shift to lower bias voltages when the temperature is raised. For example, $V_{u}$ is $6.50 \mathrm{~V}$ for $T=295 \mathrm{~K}$ and $10.98 \mathrm{~V}$, for $T=4.5 \mathrm{~K}$, respectively. Remarkably, the threshold voltage shift is almost linear over the wide temperature range from $T=4.5 \mathrm{~K}$ to $295 \mathrm{~K}$.

For a better comparison, the relative threshold voltage shift, defined by $\Delta V_{\alpha}=V_{\alpha}(4.5 \mathrm{~K})-$ $V_{\alpha}(T)$, is shown in 3B. Despite the the significant different between the corresponding currents, i.e. $I_{t} \sim \mu \mathrm{A}$ and $I_{p(v)} \sim \mathrm{mA}$, all three threshold voltages shift nearly identically with temperature within ranges of $V_{\alpha}(4.5 K)-V_{\alpha}(295 K)=4.050,4.592$ and $4.415 \mathrm{~V}$ for $V_{d}, V_{t}$ and $V_{u}$, respectively. Furthermore, a temperature sensitivity of $S=\frac{\Delta V_{\alpha}}{\Delta T}=19.2 \mathrm{mV} / \mathrm{K}$ was determined from a linear fit of all three threshold voltages. Also, 3B shows the precision ef the nano-thermemeter temperature uncertainty $\delta T$ obtained by measuring the standard deviation of the threshold voltage for each temperature and the corresponding sensitivity. The temperature uncertainty is defined by the ratio of the experimental detection setup noise floor $\sigma$ and the sensitivity, i.e. $\delta T=\sigma / S$. The noise floor is dominated by current/voltage fluctuations and the main noise source in the thermometer is shot noise. The precision displays a constant value of $5 \mathrm{mK}$ within the temperature range of $T=295 \mathrm{~K}$ to $40 \mathrm{~K}$. Below $T=40 \mathrm{~K}$, the precision increases as a result of the reduced sensitivity.

One can relate the observed large threshold shift to a temperature dependent screening in the RTD. According to Ref., ${ }^{21}$ the electron density $n_{a c}$ is temperature independent, while the temperature dependence of $n_{i}$ can be calculated as $^{22}$

$$
n_{i}=m \frac{k_{B} T}{\pi \hbar^{2}} \ln \left[1+\exp \left(\frac{\mu-E_{i}}{k_{B} T}\right)\right]
$$

with $k_{B}$ being the Boltzmann constant, $T$ the temperature, $m$ the effective mass, $E_{i}$ the energy of the localized state, and $\mu$ the electrochemical potential.

Using charge conservation and neutrality, $\Pi^{+} l_{1}-\left(n_{a c}+n_{i}\right)+N_{D}^{+}\left(l_{N}+l_{2}\right)=0$, with the 
convention that $n_{a c}, n_{i}, \Pi^{+}$, and $N_{D}^{+}$have a positive sign. Therefore, the RTD voltage drop $V_{r t d}$ is given by

$$
\begin{array}{r}
V_{r t d}=V_{d b}-\frac{n_{a c}}{\varepsilon}\left(l_{N}+l_{2}\right)+\frac{N_{D}^{+}}{2 \varepsilon}\left(l_{N}+l_{2}\right)^{2} \\
-\frac{\Pi^{+}}{\varepsilon} l_{1} l_{d b}+\frac{n_{i}}{\varepsilon}\left(l_{1}+l_{a}+l_{d b}+l_{N}\right) \\
-\frac{n_{i}-\Pi^{+} l_{1}}{\varepsilon}\left(l_{1}+l_{a}+l_{d b}+l_{N}+l_{2}\right)-\frac{\Pi^{+} l_{1}^{2}}{2 \varepsilon},
\end{array}
$$

where $V_{d b}=-\frac{n_{a c}-\Pi^{+} l_{1}}{\varepsilon} l_{d b}$, is the voltage drop across the double barrier. To model the temperature dependence of the RTD threshold voltage, we considered that the charge accumulated before the double barrier, $n_{a c}=N_{D}^{+}\left(l_{N}+l_{2}\right)$, is independent on temperature ${ }^{21}$ and $n_{i}=\Pi^{+} l_{1}$. In this way, changes of the charge concentration $n_{i}$ also affect the size of the depletion region with positively charged donor concentration $\Pi^{+}$. For a constant transmission probability of the RTD, e.g. at the peak current, the voltage at the DBS, $V_{d b}$, is constant. Using 5 and the neutrality condition constraints, one obtains

$$
\begin{aligned}
V_{r t d}=-\left|V_{d b}\right|- & \frac{V_{d b}^{2} \cdot \varepsilon}{2 N_{D}^{+} l_{d b}^{2}}+\frac{n_{i}^{2}}{2 \varepsilon}\left(\frac{1}{\Pi^{+}}-\frac{1}{N_{D}^{+}}\right) \\
& +\frac{n_{i}}{\varepsilon}\left(l_{N}+l_{a}-l_{d b} \frac{\left|V_{d b}\right|}{N_{D}^{+} l_{d b}^{2}}\right) .
\end{aligned}
$$

Note, that $V_{r t d}<0$ for $n_{i}=0$. For high doped donor density and $\Pi^{+} \simeq N_{D}^{+}$, the third term in $6\left(\sim n_{i}^{2}\right)$ can be neglected with respect to the linear term. Thus, if $l_{N}+l_{a}-l_{d b} \frac{\left|V_{d b}\right|}{N_{D}^{+} l_{d b}^{2}}>0$, the linear term is positive. This condition can be attained for large enough values of $\left(l_{N}+l_{a}\right)$ and/or $N_{D}^{+}$.

The last term in 6 becomes, $\Delta V \simeq \frac{n_{i}}{\varepsilon}\left(l_{N}+l_{a}-2 l_{d b}\right)$, for $\left|V_{d b}\right| \simeq 0.3 \mathrm{~V}, l_{d b}=10 \mathrm{~nm}$, and $N_{D}^{+}=10^{18} \mathrm{~cm}^{-3}$. Then, since $l_{N}+l_{a}-2 l_{d b} \simeq l_{N}$, for a constant voltage drop at the double barrier, $V_{d b}$, an increase in $n_{i}$ with temperature will lead to the voltage shift,

$$
\Delta V \simeq \frac{n_{i}}{\varepsilon} l_{N},
$$


which reduces the absolute value of $\left|V_{r t d}\right|$, i.e. $\left|V_{r t d}\left(T_{1}\right)\right|<\left|V_{r t d}\left(T_{2}\right)\right|$ for $T_{1}>T_{2}$.
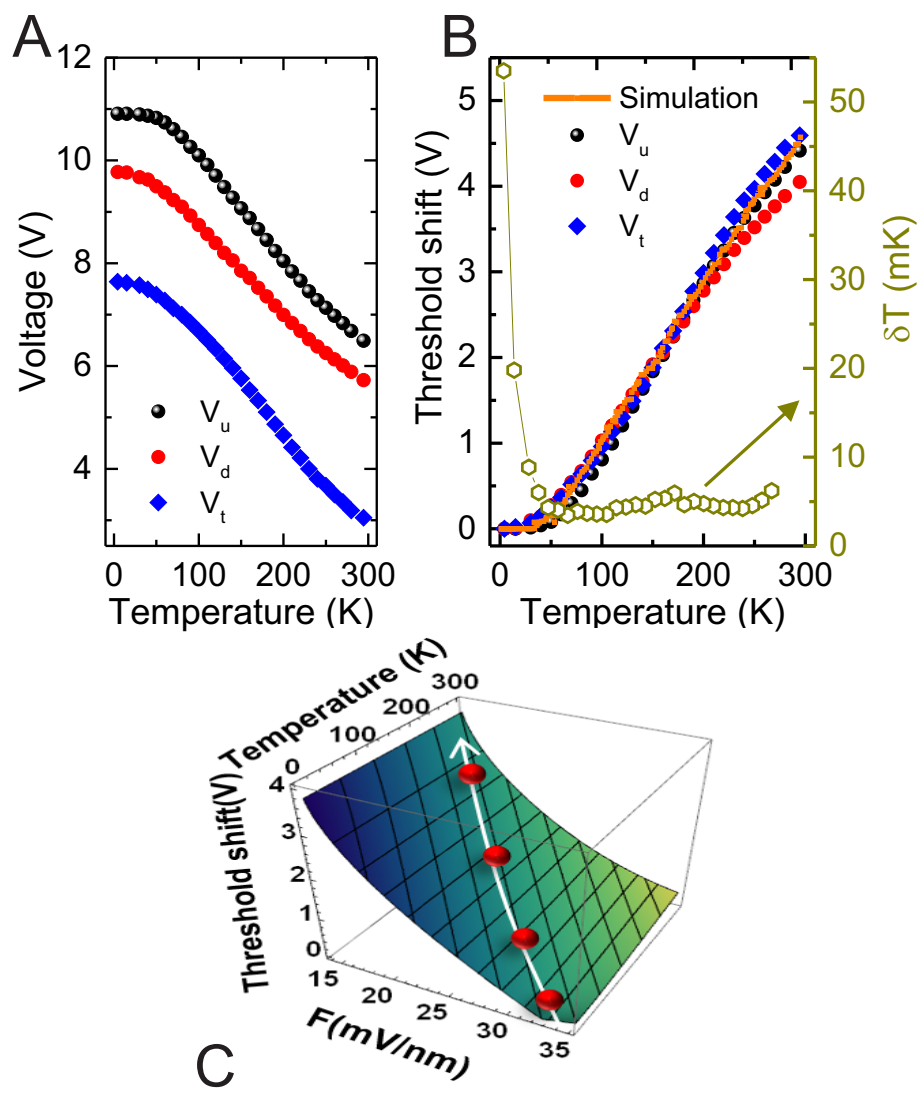

Figure 3: The electrical read-out of the nano-thermometer is provided by a reduction of the threshold voltages $V_{\alpha}$ with $\alpha=t, u, d$ for a temperature raise from $T=4.5 \mathrm{~K}$ to $295 \mathrm{~K}$. (A) Temperature dependence of the distinct voltages $V_{u}, V_{d}$ and $V_{t}$, with $I\left(V_{t}\right)=100 \mu \mathrm{A}$. (B) Here, the corresponding relative shifts of the threshold voltages, $\Delta V_{\alpha}$ are shown and the precision of the nano-thermometer. In total, there is a shift of about $4.5 \mathrm{~V}$ with an almost linear slope of $19.2 \mathrm{mV} / \mathrm{K}$. (C) Calculated threshold voltage shift as a function of temperature and applied electric field.

A temperature raise increases the charge density $n_{i}$ which, in turn, reduces the total voltage drop $V_{r t d}$. This condition is required to drive the RTD at a constant transmission probability with a DBS voltage drop $V_{d b}$. Thus, more electrons are confined in the triangular shaped well, which is formed at the GaInNAs/GaAs interface and modulated by the potential $-|e| F z$, where $F$ is an electric field locally produced by an external bias and charge redistribution along the structure, as depicted in $1 \mathrm{~B}$ and $\mathrm{D}$. A temperature raise results in additional electron accumulation at the interface, which screens the electric field and causes a reduction of $E_{i}$ which increases the electron density. By simplifying the confined level at 
the triangular well formed at the junction, its energy value with respect to the drain contact is given by $\mu-E_{i} \simeq V_{0}-\left(\frac{\hbar^{2}}{2 m}\right)^{1 / 3}\left(9 / 8 \pi e F_{\text {eff }}\right)^{2 / 3}$, where $V_{0}=230 \mathrm{meV}$ is the depth of the triangular potential with respect to the drain and $F_{\text {eff }}$ the corresponding effective electric

field. $F_{\text {eff }}$ is given by $F_{e f f}=F-\frac{e \cdot n_{i}}{2 \varepsilon}$ and accounts for the electric field screening of the temperature-dependent charge density $n_{i}$, which reduces the field $F . F_{\text {eff }}$ and 4 set a transcendental equation that can be numerically solved for $n_{i}$. These values were introduced into the voltage shift of 7 that was plotted in 3C. Thus, to observe the large shift $\Delta V$ it is essential to have a wide GaInNAs quantum well with $l_{N}=160 \mathrm{~nm}$ and high doping level $n_{i}$. There is however a limiting factor to the size of the GaInNAs layer. For a wide quantum well, the sample quality is degraded due to unavoidable crystal defects within GaInNAs and the resistance is strongly enhanced. When this resistance effect becomes dominant, it limits the performance of the device. The shift of the RTD threshold with temperature allows an electrical read-out of the device. The projection of the symbols on the surface in $3 \mathrm{C}$ onto the temperature-voltage plane corresponds to the theoretical curve plotted in $3 \mathrm{~B}$.

\section{Thermometric optical read-out}

Thermometric optical read-out. Additionally, the RTD nano-thermometer emits light with the GaAs bandgap energy through electron-hole pair recombination, in which holes are created by impact ionization. ${ }^{23} 4 \mathrm{~A}$ and $\mathrm{B}$ show the contrasting emission conditions for no applied bias and for a biased nano-thermometer above the $V_{u}$ resonant threshold. The streng electroluminescence (EL) is also a thermometric function of the device and two examples of the temperature resolved spectra are displayed in $4 \mathrm{C}$.

The EL signal from the RTD can be analyzed by either the spectral maximum or the integrated intensity. Since the RTD emits light with the GaAs bandgap energy with intensity that peaks at the resonant current condition, the temperature information read-out can also be attained optically and remotely via via EL, as shown in 5A and B. 5A illustrates the position of the spectral maximum, obtained experimentally, corresponding to the GaAs 

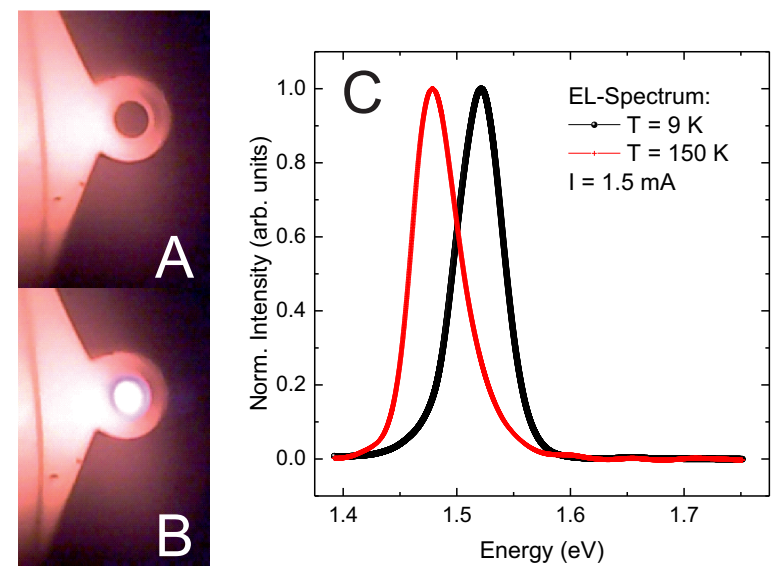

Figure 4: The optical read-out of the nano-thermometer is provided by a strong the electroluminescent (EL) emission. (A) Top-view photograph of the nano-thermometer for zero bias voltage. The Au-ring contact is centered on top of the RTD mesa structure. (B) Topview photograph of the nanothermometer biased above $V_{u}$ : a streng-EL-signal is observed from the RTD-mesa. (C) Normalized EL-Spectra for Temperatures $\mathrm{T}=9 \mathrm{~K}$ (black) and $\mathrm{T}=150 \mathrm{~K}$ (red). The EL emission can be ascribed to the GaAs drain region of the RTD nano-thermometer.

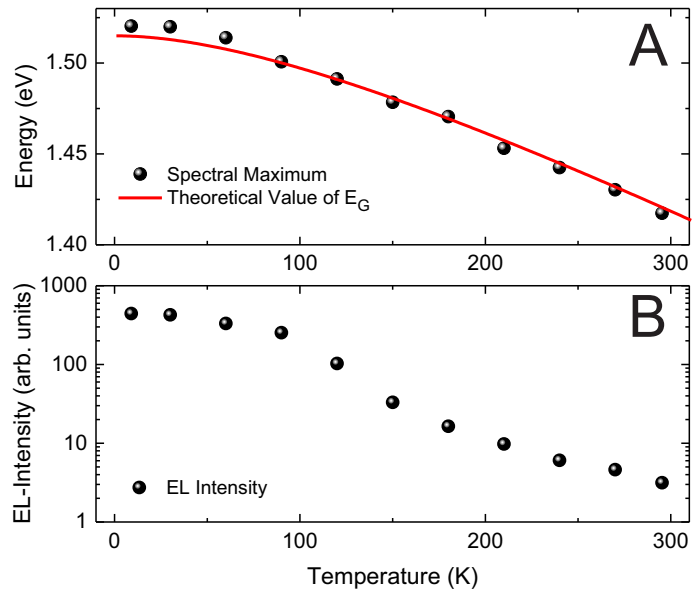

Figure 5: (A) Energy of the spectral maximum of the electroluminescence signal. The theoretical bandgap energy of GaAs according to the Varshni model is also shown. (B) The optical read-out by the integrated EL intensity can be complementary used to detect the temperature at the junction with three orders of magnitude increment. 
energy gap, as shown by the theoretical points after the Varshni model ${ }^{24}$ as,

$$
E_{g}=E_{0}-\alpha T^{2} /(T+\beta)
$$

with $E_{0}=1.5216 \mathrm{eV}, \alpha=8.871$, and $\beta=572 .{ }^{24}$ This agreement can be used for calibration purposes. Under operation, the RTD generates heat, which can be minimized under low power configuration, i.e. read-out of $V_{t}$ for a small threshold current, $I_{t}$. Yet, as noted in 5A, obtained at the condition of resonant current, no sensitive self-heating effect seems to be apparent, since the environmental temperature coincides with the one expected from the spectral position of the EL-emission.

Beside the thermometric energy shift, the emission intensities can also be used for readout. The relative emission strength, obtained at working configuration with constant current flow, varies within a three order range, as spotted in 5B. As the threshold voltage is shifted towards lower values, the electrons injected from the resonant tunneling structure have less energy when reaching the drain and therefore a reduced probability to undergo an impact ionization process. Thus, fewer holes are created and subsequently the temperature increase leads to a reduction of the EL intensity as shown in 5B.

Comparison of the thermometric read-outs. The figure of merit of different thermometers is the relative sensitivity $S_{\text {rel, }}$ which displays the percentage change of the read-out output versus temperature $1,10,25$. $S_{x e l}$ allows comparing the different read-out modes of the RTD nano-thermometer. Either electrically via the read-out of the threshold voltages, $V_{\alpha}$ or optically via the read-out of the spectral position and intensity of the EL signal ( $E_{M}$ and $I_{E L}$, respectively). The relative sensitivities for the different read-out outputs are given by dividing the absolute sensitivity by the absolute output parameter,

$$
S_{r e l}=\frac{\partial V_{\alpha}}{\partial T} / V_{\alpha}, \frac{\partial I_{E L}}{\partial T} / I_{E L}, \frac{\partial E_{M}}{\partial T} / E_{M} .
$$

The temperature dependence of $S_{r e l}$ is displayed in Figure 6A for the electrical read-out, 
and in Figure 6B for the optical read-out. For the electrical read-out the threshold voltage, $V_{\text {t }}$ shows the highest relative sensitivity with a maximum of $S_{x e l}=0.5 \% \mathrm{~K}^{-1}$ at $\mathrm{T}=220 \mathrm{~K}$. For $V_{u}$ and $V_{d}$ the relative sensitivities are about half the value for $\mathrm{Vt}$ (because $\left.V_{u}, V_{d} \geq V_{t}\right)$. For temperatures below $\mathrm{T}=50 \mathrm{~K}, S_{r e l}$ is reduced due to the decreasing absolute sensitivity. In addition to the highest relative sensitivity the read-out of $V_{t}$ also offers a low power operation in combination with the lowest noise floor and smallest temperature uncertainty (see Figure 3B). By operating the device in a constant voltage mode with the current, $I_{2}$ serving as read-out parameter, the relative sensitivity $S_{\text {rel }}=(\partial I / \partial T) / I$, can be further enhanced. The relative sensitivity of the constant-voltage mode is depicted in Figure 6A as black stars for bias voltage $V=4.75 \mathrm{~V}$. Excellent relative sensitivities with a maximum of $S_{r e l}=6.1 \% \mathrm{~K}^{-1}$ at $\mathrm{T}=90 \mathrm{~K}$ are observed, that correspond to an enhancement of one order of magnitude compared to the threshold voltage read-out. The enhanced relative sensitivity originates from the exponential RTD current-voltage characteristics and a drawback of this operation mode is the nonlinear temperature dependence with a varying absolute sensitivity. For the optical read-out, displayed in Figure 6B, the intensity of the EL signal (black stars) exhibits the highest relative sensitivity with values up to $S_{x e l}=4.35 \% \mathrm{~K}^{-1}$ which is about two orders of magnitude larger than the maximum relative sensitivity of the position of the EL maximum, $S_{x e l}=0.035 \% \mathrm{~K}^{-1}$, shown with black spheres.

\section{Conclusions}

In summary, we have presented a novel temperature sensor concept based on the temperature dependent threshold voltage shift of RTDs with a GaInNAs/GaAs heterojunction in sequence. The temperature dependent sheet charge density and donor concentration at the interface modulate the internal electric field of the resonant tunneling structure, which results in a pronounced negative threshold voltage shift when the temperature is changed from cryogenic to room temperature. This carrier density controls the voltage drop at the DBS 


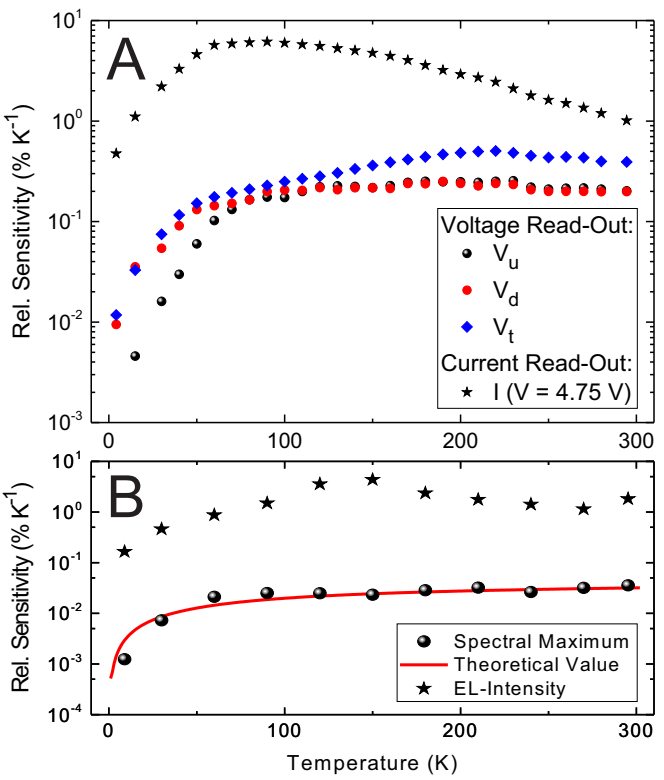

Figure 6: (A) Relative sensitivities of the electrical read-out. The voltage read-out mode displays relative sensitivities in the range of 0.25 to $0.5 \% \mathrm{~K}^{-1}$ for $\mathrm{T}=220 \mathrm{~K}$ at a constant absolute sensitivity. In the current read-out mode the sensitivity reaches values up to 6.1 $\% \mathrm{~K}^{-1}$ at $\mathrm{T}=90 \mathrm{~K}$. (B) Relative sensitivities of the optical read-out. The relative sensitivity of the EL intensity is enhanced by two orders of magnitude compared to the peak maximum position 
which, in turn, changes the transmission probability of the RTD. The temperature-threshold voltage shift relation is linear with a high response of $19.2 \mathrm{mV} / \mathrm{K}$, which promotes these structures as sensitive thermometers. Concomitant optical thermometric parameters of the strong-EL of this nano-thermometer were presented and characterized. These properties guarantee accurate electrical and optical read-outs of the sensing operation and a possible on-chip realization.

\section{Methods}

Growth and fabrication. The sample was grown by molecular beam epitaxy on (100)-oriented, silicon-doped GaAs substrates with no offcut. We used a 3 inch EIKO MBE system with standard solid sources, aside from $\mathrm{N}$ which was provided as atomic species by a RF plasma source. To reduce ion induced damage of the growth surface, deflection plates with an applied bias were used to redirect charged $\mathrm{N}$ atoms from their direct way to the growth Surface. The growth temperature of the nitrogen containing layers was $370{ }^{\circ} \mathrm{C}$, and else $595^{\circ} \mathrm{C}$. As was supplied as As by a valved cracking cell. The As/group-III flux ratio was $\approx 25$ with an As-beam equivalent pressure of $2.0 \times 10^{-5}$ torr. After the growth process, the bottom AuGe/Ni/Au contact was evaporated and alloyed via a rapid thermal annealing process with $380^{\circ} \mathrm{C}$ for 30 s. The samples were then processed using a standard PMMA positive resist electron beam lithography process with a $\mathrm{BaF}_{2} / \mathrm{Cr}$ etching mask and a low power electron cyclotron resonance dry chemical etching process with an argon to chlorine ratio of about 10. Sample planarization was realized with Benzocyclobutene. The ring shaped Ti/Pt/Au top contacts were fabricated by a second standard PMMA positive resist electron beam lithography process.

Electro-optical readout. The electro-optical transport measurements were conducted by mounting the sample in an Oxford instruments microscopy cryostat - Microstat MO. The electrical bias and read-out was conducted using a high precision voltage source (Hewlett 
Packard 3251 Universal Source) and a digital multimeter (Keithley 2000 Digital Multimeter).

The electroluminescence data was collected through a microscope objective (0.4NA) and directed into a Jobin-Yvon Triax monocromator(focal length $500 \mathrm{~mm}$ ) using a set of lenses and mirrors. The monochromator has a spectral resolution of $0.05 \mathrm{meV}$ and an attached nitrogen-cooled Silicon-CCD (Jobin-Yvon, type Spectrum 1) was used as detector.

\section{Acknowledgement}

The authors are grateful for financial support by the BMBF via via national project EIPHRIK (FKZ: 13N10710), the European Union (FPVII (2007-2013) under grant agreement no. 256959 NANOPOWER and no. 318287 LANDAUER), and the Brazilian Agencies FAPESP (2013/24253 - 5, 2012/13052 - 6, and 2012/51415 - 3), CNPq and CAPES. The expert technical assistance by M. Emmerling and S. Handel is gratefully acknowledged.

\section{References}

(1) Brites, C.; Lima, P.; Silva, N.; Millan, A.; Amaral, V.; Palacio,F.; Carlos, L. Thermometry at the nanoscaleNanoscale. Nanoscale 2012, 4, 4799-4829.

(2) Childs, P.; Greenwood, J.; Long, C. Review of emperature measurementTemperature Measurement. Rev. Sci. Instrum. 2000, 71, 2959.

(3) Sadat, S.; Tan, A.; Chua, Y. J.; Reddy, P. Nanoscale Thermometry Using Point Contact Thermocouples. Nano Lett. 2010, 10, 2613-2617.

(4) Mecklenburg. M.: Hubbard, W. A.: White E. R.: Dhall, R.: Cronin, S. B. Aloni, S.i Regan, B. C. Nanoscale Temperature Mapping in Operating Microelectronic Devices. Science. 2015, 34\%, 629-632.

(5) Huang, H.; Delikanli, S.;. Zeng, H; Ferkey, D. M.; Pralle, A. Remote eontrol of 
ion channels and neurons through magnetic-field heating of nanoparticles. Control of Ion Channels and Neurons through Magnetic-Field Heating of Nanoparticles. Nature NanotechnologyNat. Nanotechnol. 2010, 5, 602-606.

(6) Okabe, K.; Inada, N.; Gota, C.; Harada, Y.; Funatsu, T.; Uchiyama, S. Intracellular temperature mapping with a fluorescent polymeric thermometer and fluorescence lifetime imaging microscopy. Temperature Mapping with a Fluorescent Polymeric Thermometer and Fluorescence Lifetime Imaging Microscopy. Nature Nat. Commun. 2012, 3, 705 .

(7) Lu, W.; Lieber, C. M. Nanoelectronics from the bottom up. Bottom up. Nature MatNat. Mater. 2007, 6, 841-850.

(8) Wong, H.; Iwai, H. On the sealing issues and high-Scaling Issues and High- $\kappa$ replacement of ultrathin gate dielectries for nanoseale MOS transistors. Replacement of Ultrathin Gate Dielectrics for Nanoscale MOS Transistors. Microelectronic EngineeringMicroelectron. Eng. 2006, 83, 1867-1904.

(9) Giazotto, F.; Heikkila, T. T.; Luukanen, A.; Savin, A.M.; Pekola,J. P. Opportunities for mesoscopies in thermometry and refrigerationMesoscopics in Thermometry and Refrigeration: Physics and applicationsApplications. Rev. Mod. Phys. 2006, 78, 217274 .

(10) Pinol, R.; Brites, C. D. S; Bustamante, R.; Martinez, A.; Silva, N. J. O.i Murillo, J. L.i Cases, R.: Carrey J.: Estepa, C.: Sosa, C.; Palacio, F.: Carlos, L. D.; Milln. A. Joining Time-Resolved Thermometry and Magnetic-Induced Heating in a Single Nanoparticle Unveils Intriguing Thermal Properties. ACS Nano 2015, 9, 3134-3142.

(11) Kim, W.; Wang, R.; Majumdar, A. Nanostructuring expands thermal limits.Expands Thermal Limits. NanotodayNano Today 2007, 2, 40-47. 
(12) Liu, Z.; Bando, Y.; Hu, J.; Ratinac, K.; Ringer, S. A novel method for practical temperature measurement with earbon nanotube nanothermometers Novel Method for Practical Temperature Measurement with Carbon Nanotube Nanothermometers. Nanotechnology 2006, 17, 3681-3684.

(13) Crossno J.i Liu, X.: Ohki, T. A.i Kim, P.i and Fong, K. C. Development of High Frequency and Wide Bandwidth Johnson Noise Thermometry. Appl. Phys. Lett. 2015 106, 023121.

(14) Spietz, L.; Lehnert, K. W.; Siddiqi, I.; Schoelkopf, R. J. Primary Electronic Thermometry Using the Shot Noise of a Tunnel Junction. Science 2003, 300, 1929-1932.

(15) Hartmann, F.; Langer, F.; Bisping, D.; Musterer, A.; Höfling, S.; Kamp, M.; Forchel, A.; Worschech, L. GaAs/AlGaAs resonant tunneling diodes Resonant Tunneling Diodes with a GaInNAs absorption layer for telecommunication light sensingAbsorption Layer for Telecommunication Light Sensing. Appl. Phys. Lett. 2012, 100, 172113.

(16) Tsu R.; Esaki, L. Tunneling in a Finite Superlattice. Appl. Phys. Lett. 1973, 22562.

(17) Mizuta, H.; Tanoue, T. The Physics and Aplications of Resonant Tunnelling Diodes, ed. Ahmed, H.; Pepper, M.; Broers, A. (Cambridge University Press, New York, 1995), p. 26 .

(18) De Carvalho, H. B.; Brasil, M. J. S. P.; Lopez-Richard, V.; Galvao Gobato, Y.; Marques, G. E.; Camps, I.; Dacal, L. C. O.; Henini, M.; Eaves, L.; Hill, G. Electric-field inversion asymmetryElectric-Field Inversion Asymmetry: Rashba and Stark effects for holes in resonant tunneling devicesEffects for Holes in Resonant Tunneling Devices. Phys. Rev. B 2006, 74, 041305.

(19) Schulman, J. N.; De Los Santos, H. H.; Chow, D. H. Physics-based RTD current-voltage equation.Physics-Based RTD Current-Voltage Equation. IEEE Electron Device Letters, IEEELEtt. 1996, 17(5), 220-222. 
(20) Büttiker, M. Coherent and sequential tunneling in series barriers. Sequential Tunneling in Series Barriers. IBM Journal of Research and Development. J. Res. Dev. 1988, 32(1), 63-75.

(21) Bar-Joseph, I.; Gedalyahu, Y.; Yacoby, A.; Woodward, T. K.; Chemla, D. S.; Sivco, D. L.; Cho, A. Y. Temperature dependence of the resonant-tunneling process in a double-barrier diodeDependence of the Resonant-Tunneling Process in a Double-Barrier Diode. Phys. Rev. B 1991, 44, 8361.

(22) Bastard, G. Wave Mechanics Applied to Semiconductor Heterostructures, p. 163. (Les Editions de Physique, France, 1991).

(23) White, C. R. H.; Eaves, L.; Leadbeater, M. L.; Henini, M.; Hughes, D. H.; Hill, G.; Pate, N. A. The ebservation of electroluminescence-Observation of Electroluminescence in a $\mathrm{GaAs}(\mathrm{AlGa})$ As double barrier resonant tunnelling structure. Double Barrier Resonant Tunnelling Structure. Superlattice MicrostSuperlattices Microstruct. 1990, 8, 391.

(24) Varshni, Y. P. Temperature Dependence of the Energy Gap in Semiconductors Physica 1967, 34, 149-154.

(25) Wang, Z.i Arnau Carne-Sanchez, D.-A.; Brites, C. D. S.i Imaz, I.i Maspoch, D.i Rocha, J.; Carlos, L. D. Lanthanide-Organic Framework Nanothermometers Prepared by Spray-Drying. Adv. Funct. Mater. 2015, DOI: 10.1002/adfm.201500518. 